International Journal of VLSI design \& Communication Systems (VLSICS) Vol.7, No.3, June 2016

\title{
DESIGNING AN EFFICIENT APPROACH FOR JK AND T FLIP-FLOP WITH POWER DISSIPATION ANALYSIS USING QCA
}

\author{
Shraddha Pandey, Sonali Singh and Subodh Wairya \\ Department of Electronics, \\ Institute of Engineering and Technology, Lucknow, 226021, U.P, India
}

\begin{abstract}
QCA (Quantum Dot Cellular Automata) is an emerging and pioneer technology, which is a paradigm for computing with interacting quantum dots. Many eminent researchers have well thought of eloquent work in the existing areas of the sequential circuit. However, this paper proclaims three new approaches to design $J K$ and $T$ flip-flop. Since flip-flops and memory design are the crucial building blocks of digital circuits, therefore we concern the underlying principle of fundamental design of JK and T flip-flop and then work out to model the new structure favorable with the forthcoming excellence required. This new concept places elsewhere the need of using feedback path in flip flop design. Also two algorithms have been shown for explanatory purpose. The proposed structure is able to establish the validity and genuineness than earlier design. By using the proposed T flip-flop, a 2-bit and 3-bit counter is also designed in the paper. The simulation result of the proposed design proves their vigorousness and correctness in the output.
\end{abstract}

\section{KEYWORDS}

Quantum-dot cellular automata (QCA), Level triggered flip-flop, Counter, Kink energy, Thermal hot spots, Nanometre scale.

\section{INTRODUCTION}

According to Moore's law, it is observed that over the history of computing hardware the number of transistors in a dense integrated circuit has doubled approximately every two years. But computing hardware the number of transistors in a dense integrated circuit has doubled approximately every two years. But at nano regimes, computation is substantially different from conventional VLSI. The current CMOS technology is going to face a scaling limit in deep nanometres technologies. In order to overcome the physical limitation of MOSFET based VLSI circuit design, such as tunnelling currents, sub-threshold leakage, quantum effects , fabrication cost, interconnect delay, the researchers are now focusing on developing a new technology that uses quantum mechanics and cellular automata simultaneously. And thus, QCA (Quantum Dot Cellular Automata) is the best replacement of the CMOS based VLSI design. It is a novel nanotechnology which promises extra low power, extremely dense and very high-speed structure for designing any arbitrary function at the nanoscale [1]. In 1993, Lent.et al. proposed a physical implementation of an automation using quantum-dot cells.

The automation quickly gained popularity and it was first fabricated in 1997 [2]. With the use of QCA technology, new physical phenomena such as Coulombic interactions can be exploited. As DOI : $10.5121 /$ vlsic.2016.7303 
International Journal of VLSI design \& Communication Systems (VLSICS) Vol.7, No.3, June 2016

there is no electrical current in QCA, signal propagation takes place according to coulomb's law. For this, a quantum cell is used which is much smaller than transistors and yield better results in speed. Hence, the electronic devices based on this can become denser. Due to these features researchers have done much exploration in digital electronics. This field is divided into two categories; combinational and sequential circuits. In QCA, both the circuits are implemented efficiently. By using the same Boolean logic functions used in the conventional CMOS circuits, the combinational circuits are directly implemented. But in order to design sequential circuits, the truth table is identified and then the Boolean logic equations are derived. Finally, the sequential circuit is designed by using the derived equations. A number of QCA based logic circuits with remarkable advantage have been proposed with the utilization of QCA. A number of combinational circuits such as adders [3], ex-or gate and multiplexer circuit [4], decoders [5] are proposed. However sequential circuit design in QCA is still in its inception and limited studies are reported, such as memory circuits $[6,7]$. Using QCA memory cells to implement sequential elements, however, would be difficult in terms of hardware and latency [8], so the design and application of RS, D, JK and T flip-flop have gained much attention. This paper consists of 6 parts. Part 2 includes the basic concept of QCA and the fundamental circuits used to design any complex circuit. We have introduced the clocking schemes used in QCA in the same part. In part 3 , a state of the art is shown on the fundamental designs and then the proposed circuits of JK and T flip-flop architectures are shown which prove its benefits in total consumed area and the number of cells used. In the same section, we have discussed a novel approach to design a T flipflop and its algorithm. Part 4 reveals the application of proposed flip-flops in counter design. Part 5 elaborates power dissipation analysis of the proposed circuit and the simulation result of all the proposed circuits is demonstrated. Finally, the paper is concluded in part 6.

\section{QCA PRELIMINARIES}

In this section, the building unit i.e. QCA cell, general logic, and gates in QCA are investigated in the order of QCA cell, QCA wires, majority gates, inverter, AND-OR gates.

\subsection{QCA Cell}

The primitive cell in QCA technology, as shown in Fig.1 (a), is a square-shaped cell. It contains four quantum dots at each corner of the cell. The quantum dot normally contains two electrons in each cell, which occupy two dots of the cell. Due to the Coulombic repulsion between the electrons, the electrons tend to occupy the position which has lower repulsion force between them and electrons can occupy stable states. Hence, they occupy the diagonal position within the cell. There are two methods possible for the placement of electrons as shown in Fig.1 (b). Therefore, two polarities are possible. If the electrons occupy the position as shown in Fig.1 (b) then it corresponds to polarization "1" i.e. logic 1 and if is arranged as shown in Fig.1(c) then it is in polarization "-1" i.e. logic 0 states. These 2 extra mobile electrons can quantum mechanically tunnel between dots, but not cells.

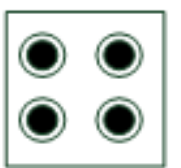

(a) QCA cell empty

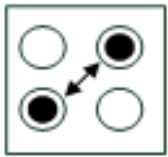

(b) 1 polarity

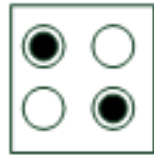

(c) 0 polarity

Fig.1 QCA cell 
International Journal of VLSI design \& Communication Systems (VLSICS) Vol.7, No.3, June 2016

Hence unlike conventional digital circuits in which information is passed by the flow of electric current, QCA operates by the Coulombic interaction that connects the state of one cell to its neighbor cell state.

\subsection{QCA wire and types}

In order to transmit the information from one cell to another, a QCA wire is needed. The wire consists of a chain of cells where the cells are also coupled to each other. The logic values are passed from one cell to the next cell due to the coulomb interaction. The polarization of the input cell is traveled down the wire. As a result, the arranged system attempt to settle down to a ground state. Due to electron repulsion if the polarization of one cell changes, it enforces its adjacent cells to change its state as shown in Fig.2.

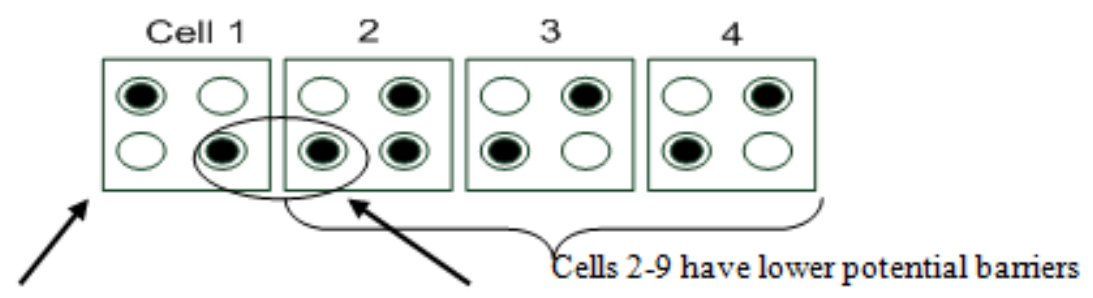

Cell $1=$ Input cell $\quad$ Cell 2 switches its polarization due to Coulombic interaction

Fig.2 QCA Wire

The way of placing quantum dots in the square cell make it possible to have two types of orientation. The first one is called as $90^{\circ}$ wire and the second is $45^{\circ}$ as shown in Fig.3(a) and 3(b) in which every other cell with opposite polarity is put together.

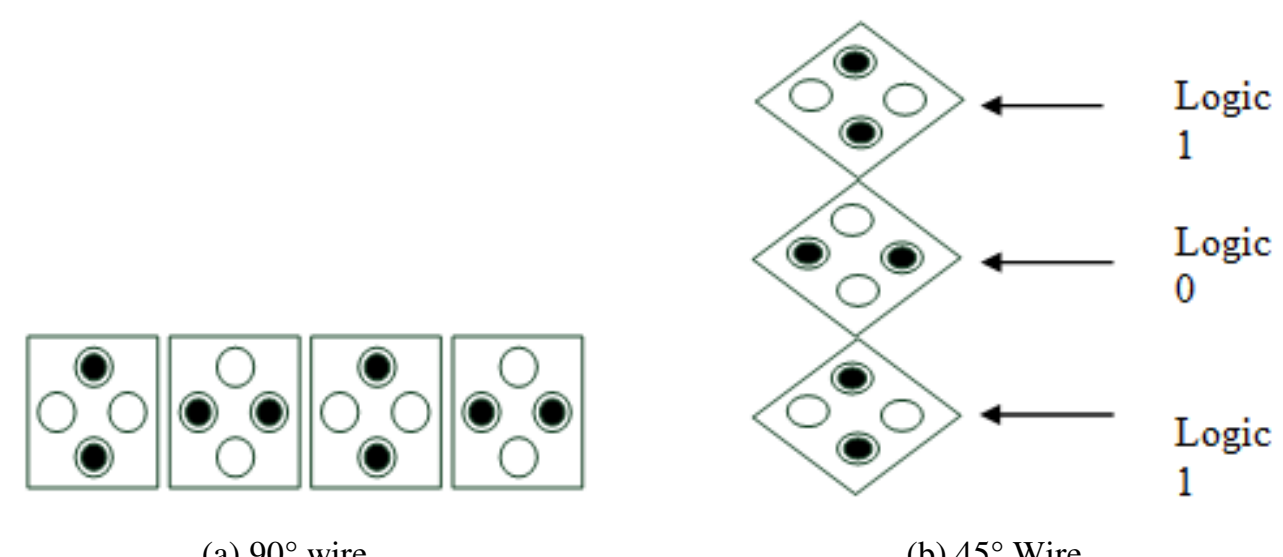

Fig.3 QCA wire types

\subsection{Majority gate}

Majority gate is one of the fundamental gates of QCA. It is shown in Fig.4 which is a 3-input majority function. The output is simply the majority of the 3 inputs applied. Assuming the inputs is $\mathrm{A}, \mathrm{B}$, and $\mathrm{C}$, the Boolean function of the majority gate is, 
International Journal of VLSI design \& Communication Systems (VLSICS) Vol.7, No.3, June 2016

$$
\mathrm{M}(\mathrm{A}, \mathrm{B}, \mathrm{C})=\mathrm{AB}+\mathrm{AC}+\mathrm{BC}
$$

Now if we fix the polarization of one input to logic " 0 ", say B, then equation (1) can be equated as follows:

$$
\mathrm{M}(\mathrm{A}, 0, \mathrm{C})=\mathrm{A} \cdot 0+\mathrm{AC}+0=\mathrm{AC}
$$

Hence equation (2) is simply the AND operation between A and C input. Similarly if we put the input B value equals to " 1 " then,

$$
\mathrm{M}(\mathrm{A}, 1, \mathrm{C})=\mathrm{A} \cdot 1+\mathrm{AC}+1 . \mathrm{C}=\mathrm{A}+\mathrm{C}+\mathrm{AC}=\mathrm{A}(1+\mathrm{C})+\mathrm{C}=\mathrm{A}+\mathrm{C}
$$

Equation (3) is an OR operation between A and C. Thus by changing the value of any of the input, the 3-input majority gate can function as AND gate and OR gate.

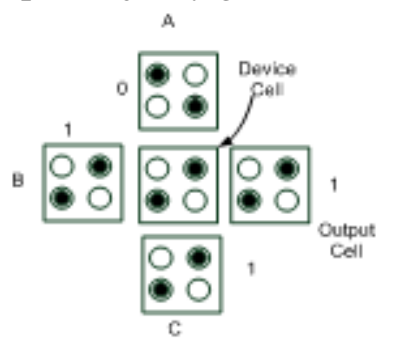

Fig.4 Majority Gate Structure [9]

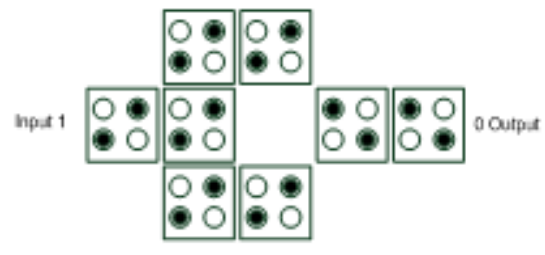

Fig. 5 (a) Inverter

\subsection{Inverter gate}

There are multiple ways to do it in the QCA paradigm that one is shown in Fig.5.In fig. (a), the signal comes from the left, splits into two parallel wires, and is inverted at the point of convergence [11]. The other arrangement used to make inverter chain as shown in Fig.5 (b) and 5 (c).

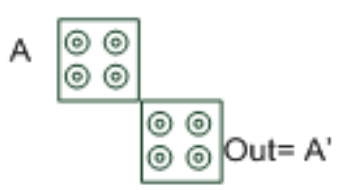

(b)

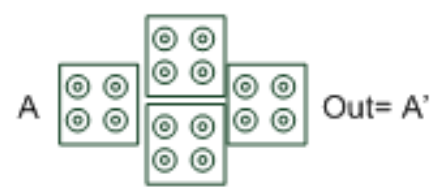

(c)

Fig.5 (a), (b), (c) Three different way to make inverter in QCA

However, placement of two cells such that they approach via only one corner leads to switch from -1 logic to +1 logic or vice versa as shown in Fig.5(b).

\subsection{QCA clocking scheme}

In VLSI design, the function is controlled by a clock signal which is inherent to the technology used. In contrast to this, QCA exhibit clocking scheme as the most fundamental concept to understand. As we know there is no flow of any current signal in the propagation of information in QCA, this does not actually means that power dissipation must be zero. Clocking in QCA not 
International Journal of VLSI design \& Communication Systems (VLSICS) Vol.7, No.3, June 2016

only confer control of information flow but also true power gain in QCA [10]. The signal energy forfeited to the environment is restored by the clock used.

Clocking in QCA is accomplished by two types of switching methods for its operation: abrupt switching and adiabatic switching. In abrupt switching, the inputs changes suddenly and the circuit can be in some excited state; consequently, the QCA circuit is relaxed to ground state by dissipating energy to the environment [11]. This inelastic relaxation is ungoverned and the QCA circuit may inscribe a metastable state that is deduced by a local, rather than a global energy ground state. Therefore, adiabatic switching is usually preferred; in adiabatic switching, the system is always kept in its instantaneous ground state [11].

After applying the clock signal, the electrons may either pushed to the four corner dots or pull them into the two middle dots. When the electrons are in the middle dots, the cell is said to be in the "null" state and when the electrons are in the four corner dots, the cell is in an active state. The cell in an active state is used to represents binary " 0 " and "1" values as shown in fig.1. This clocking scheme (which was recommended in [11] consists of four phases: Switch, Hold, Release, and Relax, as shown in Fig.6 (a).

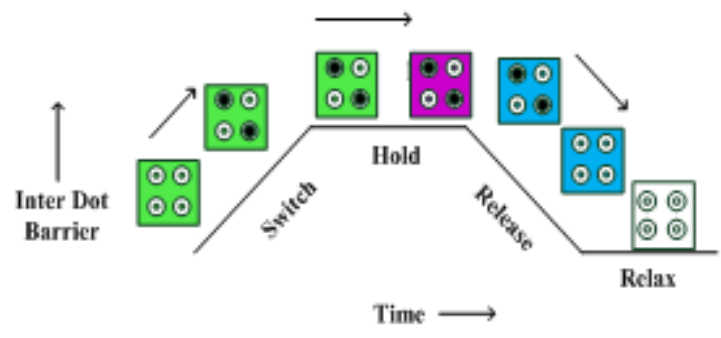

(a) Four phase clocking

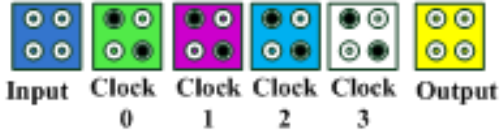

(b) Switching of signal

Fig.6 Clocking in QCA

All cells in a zone are controlled by the same clock signal. Cells in each zone perform a particular calculation. During the Relax phase, the electrons are pulled into the middle dots, and the cell is in "null" state. During the Switch phase, the interdot barrier is leisurely raised and pushes the electrons into the corner dots, so the cell acquires a definitive polarity under the dominance of its neighbors (which are in the Hold phase). The operation of fig.6 (b) is summarized below:

- During the first clock phase, the switch phase, the inter-dot potential barriers are low. The barriers are then raised during this phase and the QCA cells become polarized according to the state of their driver (i.e. their input cell).It is in this clock phase that the actual computation occurs. By the end of this clock phase, barriers are high enough to suppress any electron tunneling and cell states are fixed.

- During the second clock phase, the hold phase, barriers are held high so the outputs of the sub-array can be used as inputs to the next stage.

- In the third clock phase, the release phase, barriers are lowered and cells are allowed to relax to an unpolarized state. 
International Journal of VLSI design \& Communication Systems (VLSICS) Vol.7, No.3, June 2016

Finally, during the fourth clock phase, the relax phase, cell barriers remain lowered and cells remain in an unpolarized state.

\section{STATE OF THE ART}

Many prominent researchers are in strong progression and building sturdily in the existing areas of the sequential circuit. However, new methods are developed in order to challenge the existing designs and promising in terms of efficiency. In QCA, the research in the sequential circuit is still unveiled and due to this many concepts to design the same logic is in current trend in this field.

A novel structure of falling edge triggered clock is shown by Xiaokuo Yang in [20]. He has suggested using edge-triggered clocking scheme in place of level triggered as used by J. Huang [13] to design S-R flip flop. He said that when we design a JK flip-flop using the concept of J. Huang, we would have a race-round condition. One of the proposed circuits of falling edge triggered clock of reference [20] is shown below.

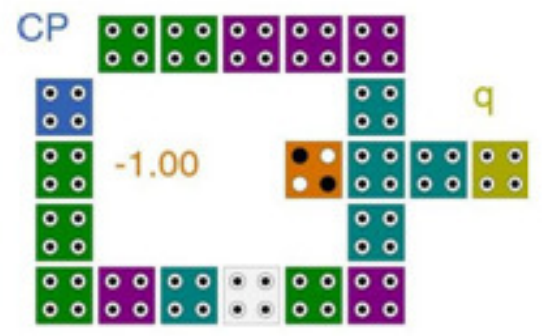

Fig.8 QCA layout of falling edge-triggered [20]

By using this circuit, JK flip-flop is constructed which uses 7 majority gates and 5 inverters. Also, 2-bit and 3-bit counters are then proposed by using the circuit of falling edge triggered JK flip-flop. By looking at the output of truth table, the output is simply AND operation between the clock input and its delayed version.

Table 1: Truth table of Fig.8 [20]

\begin{tabular}{|l|l|l|l|}
\hline $\mathrm{CP}$ & $\overline{C P}$ & CP_OLD & $\begin{array}{l}\text { OUTPUT } \\
(\mathrm{AND})\end{array}$ \\
\hline $1 \rightarrow 0$ & 1 & 1 & 1 \\
0 & 1 & 0 & 0 \\
$0 \rightarrow 1$ & 0 & 0 & 0 \\
\hline
\end{tabular}

One more researcher Lin-rong XIAO in [8] has shown method of dual edge triggered clocking scheme by using 1 latch and 1 mux only. By using this he has proposed two new QCA model of JK and D flip-flop. The output is obtained after four clock cycles.Lee Ai Lim and others in [21] have gone through all the flip-flops and its application to design counter in their paper. In his 
International Journal of VLSI design \& Communication Systems (VLSICS) Vol.7, No.3, June 2016

paper, JK flip-flop uses 4 majority gates and three inverters. However, the QCA equivalent based on the equations of Lee $\mathrm{Ai} \mathrm{Lim}$ is not competent regarding a number of cells required to formulate the objective. Hence, it is required to design a competent circuit which manifests robustness and adeptness.

Sequential circuit plays a very important role in the designing of digital circuits. By using combinational circuit only those applications are covered successfully which depends on their present state only. Sequential circuits depend not only on their present state but also on past state. To design sequential circuits, the conventional CMOS circuits are not suitable to direct translated into QCA architecture due to timing constraint of the sequential logic circuits [21]. Therefore, each sequential circuit has been derived by using the truth table and then related Boolean equation is mentioned. Each variable is named as input and output function. Then the number of required logic gates is determined.

\subsection{JK flip-flop}

A JK flip-flop is the modification of SR flip-flop. The only difference is that the intermediate state is more refined and precise than that of an SR flip-flop. The behaviour of inputs $J$ and $\mathrm{K}$ is same as the S and R inputs of the SR flip-flop. The letter J stands for SET and the letter K stands for CLEAR. The block diagram of JK flip-flop by using SR flip-flop is shown in Fig.9 and the truth table in Table 2.

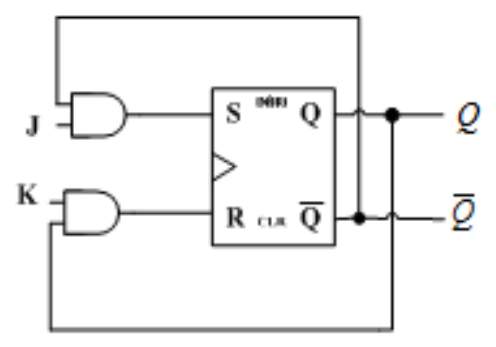

Table 2: Truth Table of JK Flip-Flop

Fig.9 JK Flip-Flop

\begin{tabular}{|l|l|l|l|}
\hline $\mathrm{J}$ & $\mathrm{K}$ & $\mathrm{Q}_{n+1}$ & STATE \\
\hline 0 & 0 & $\mathrm{Q}_{n}$ & HOLD \\
\hline 0 & 1 & 0 & RESET \\
\hline 1 & 0 & 1 & SET \\
\hline 1 & 1 & $\overline{\mathrm{Q}}_{n}$ & TOGGLE \\
\hline
\end{tabular}

Also, the JK flip-flop using NAND latch is shown in Fig.10.The QCA equivalent of crosscoupled gates is not possible. Hence, it is required to create some feedback paths to achieve this concept.

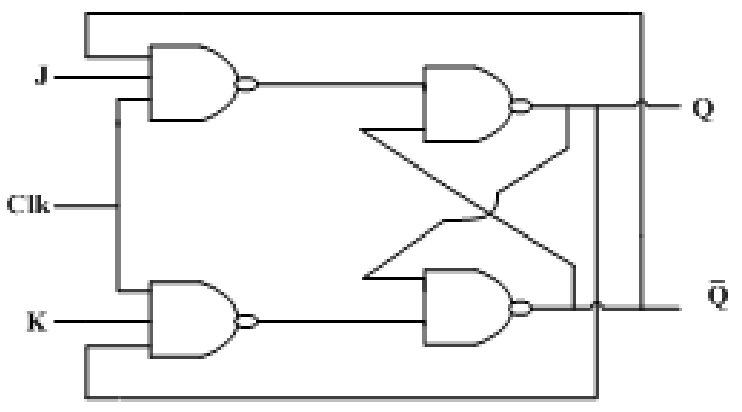

Fig.10 JK Flip-Flop Using NAND Latch 


\subsection{T flip-flop}

This is a much simplistic version of the $\mathrm{J}-\mathrm{K}$ flip-flop. Both the $\mathrm{J}$ and $\mathrm{K}$ inputs are connected together and thus are also called a single input J-K flip-flop. When the clock pulse is given to the flip-flop, the output begins to toggle which is half of the frequency of input $\mathrm{T}$. It is useful for constructing binary counters, frequency dividers, and general binary addition. The truth table is discussed below.

Table 3: TFF Truth Table

\begin{tabular}{|l|l|l|}
\hline $\mathrm{T}$ & $\mathrm{Q}_{n}$ & $\mathrm{Q}_{n+1}$ \\
\hline 0 & 0 & 0 \\
\hline 0 & 1 & 1 \\
\hline 1 & 0 & 1 \\
\hline 1 & 1 & 0 \\
\hline
\end{tabular}

One of the researcher in [21] have proposed a falling edge triggered JK flip-flop based on which a $\mathrm{T}$ flip flop is designed as shown in fig 11. The working principle of this design is somewhat similar to the proposed structure of [21] but it will use crossover technique to implement in QCA. Here a falling edge triggered clock is used as a second input to T flip-flop. The QCA equivalent block diagram is shown in Fig. 11.

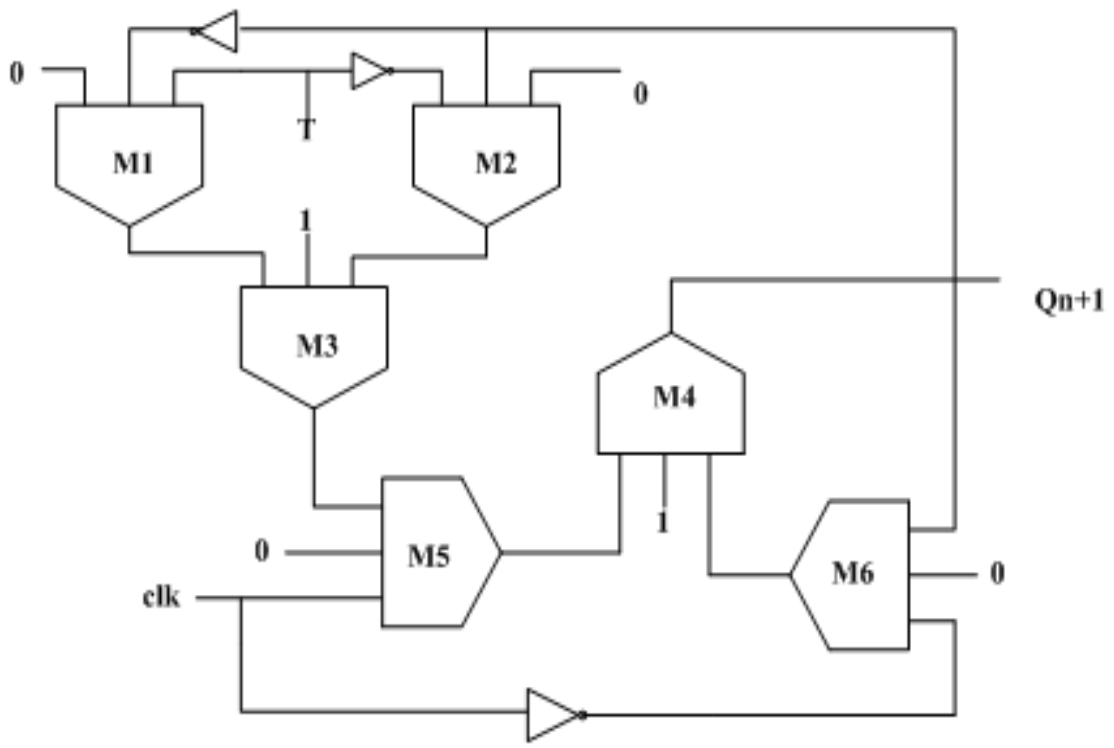

Fig.11: Approach to design T flip flop

The majority equation for the above circuit can be written as:

$$
\begin{aligned}
\mathrm{Q}_{n+1} & \left.=\mathrm{M}\left(\mathrm{M}\left(\mathrm{M}\left(\mathrm{M}(\mathrm{T}, \bar{Q} n, 0), \mathrm{M}\left(\overline{\mathrm{T}}, \mathrm{Q}_{n}, 0\right), 1\right)\right), \mathrm{CLK}, 0\right), \mathrm{M}\left(\overline{C L K}, \mathrm{Q}_{n+1}, 0\right), 1\right) \\
& =\mathrm{M}\left(\mathrm{M}\left(\left(T \cdot \bar{Q}_{n}+\bar{T} \cdot Q_{n}\right), \mathrm{CLK}, 0\right),\left(\overline{C L K} \cdot \mathrm{Q}_{n+1}\right), 1\right) \\
& =\left(T \cdot \bar{Q}_{n}+\bar{T} \cdot Q_{n}\right) \cdot \mathrm{CLK}+\overline{C L K} \cdot \mathrm{Q}_{n+1}
\end{aligned}
$$




$$
\mathrm{Q}_{n+1}=\left(\mathrm{T} \oplus Q_{n}\right) \cdot \mathrm{CLK}+\overline{C L K} \cdot \mathrm{Q}_{n+1}
$$

\subsection{Proposed Design of JK Flip-Flop}

When $\mathrm{J}$ and $\mathrm{K}$ are in HIGH state, the flip-flop switches to the complement value of previous output. The proposed JK flip-flop is controlled by using a clock and thus it is called as positive level triggered JK flip-flop because output will change its state only when the clock will be in HIGH state. The truth table of the proposed circuit is shown in Table 4.

Table.4 Truth Table of Proposed Level Triggered JK Flip-Flop

\begin{tabular}{|c|c|c|c|c|}
\hline $\mathrm{J}$ & $\mathrm{K}$ & CLOCK & \multicolumn{2}{|l|}{$\mathrm{Q}_{n+1}$} \\
\hline 0 & 0 & 0 & \multirow{2}{*}{$\left.\begin{array}{l}0 \\
0\end{array}\right\}$} & \multirow{2}{*}{ HOLD } \\
\hline 0 & 0 & 1 & & \\
\hline 0 & 1 & 0 & \multirow{2}{*}{$\left.\begin{array}{ll}0 \\
0\end{array}\right\}$} & \multirow{2}{*}{ RESET } \\
\hline 0 & 1 & 1 & & \\
\hline 1 & 0 & 0 & \multirow{2}{*}{$\left.\begin{array}{ll}0 \\
1\end{array}\right\}$} & \multirow{2}{*}{ SET } \\
\hline 1 & 0 & 1 & & \\
\hline 1 & 1 & 0 & \multirow{2}{*}{$\left.\begin{array}{l}1 \\
0\end{array}\right\}$} & \multirow{2}{*}{ TOGGLE } \\
\hline 1 & 1 & 1 & & \\
\hline
\end{tabular}

From the above truth table, we can write the expression for $\mathrm{Q}_{n+1}$ by going through the following algorithm as below:

\section{Algorithm 1: Level triggered JK flip-flop}

Input: $\mathrm{A}, \mathrm{B}, \mathrm{C}$ where

$\mathrm{A}=\mathrm{J}$

$\mathrm{B}=\mathrm{K}$;

$\mathrm{C}=\mathrm{CLOCK}$;

Output: $\mathrm{Q}_{n}, \mathrm{Q}_{n+1}$, where $\mathrm{Q}_{n}=$ previous output and $\mathrm{Q}_{n+1}=$ present output

(To set the value of inputs, we take the vector form as)

Set input $\left((\mathrm{A}, \mathrm{B}, \mathrm{C}) \&\right.$ output $\left.\left(\mathrm{Q}_{n}\right)\right)$

$\mathrm{A}=0,1$;

$\mathrm{B}=0,1$;

$\mathrm{C}=0,1$;

$\mathrm{Q}_{n}=0$;

Set variable $\mathrm{i}=0$ to $\mathrm{n}$; (for the number of combination)

\section{begin}

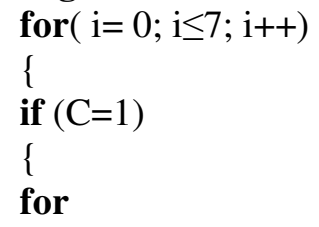


International Journal of VLSI design \& Communication Systems (VLSICS) Vol.7, No.3, June 2016
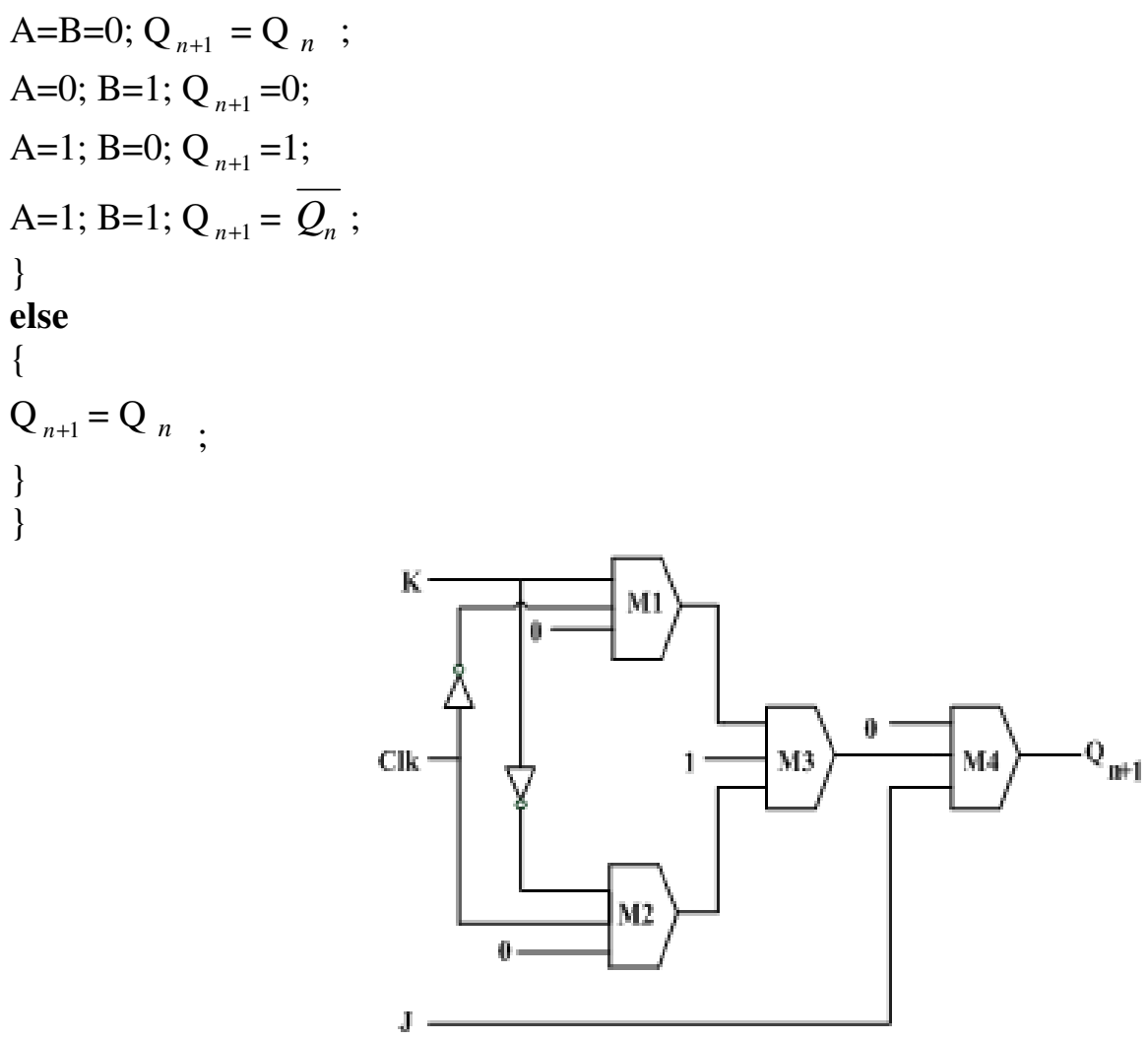

Fig. 12 (a) Block Diagram of proposed level triggered JK flip-flop

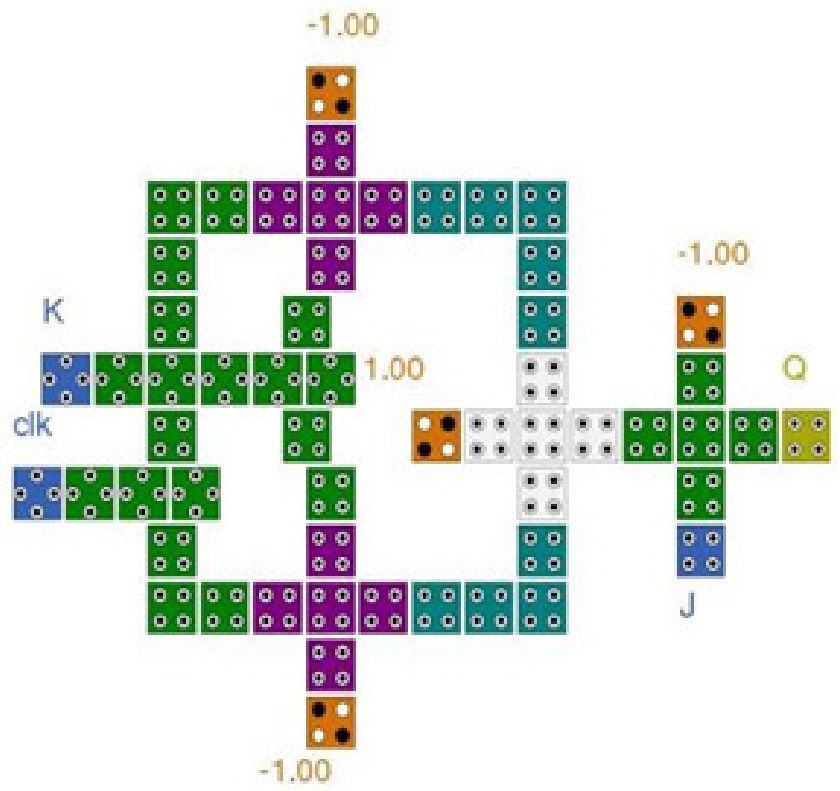

Fig. 12 (b) Layout of proposed level triggered JK flip-flop 
International Journal of VLSI design \& Communication Systems (VLSICS) Vol.7, No.3, June 2016

The majority logic equation can be derived by observing the above block diagram as follows:

$$
\begin{aligned}
\mathrm{Q}_{n+1} & =\mathrm{M}(\mathrm{M}(\mathrm{M}(\mathrm{K}, \overline{C L K}, 0), \mathrm{M}(\bar{K}, \mathrm{CLK}, 0), 1), \mathrm{J}, 0)) \\
& =\mathrm{M}(\mathrm{M}((\mathrm{K} \cdot \overline{C L K}),(\bar{K} \cdot \mathrm{CLK}), 1), \mathrm{J}, 0) \\
& =\mathrm{M}((K \cdot \overline{C L K}+\bar{K} \cdot C L K), \mathrm{J}, 0) \\
\mathrm{Q}_{n+1} & =(K \overline{C L K}+\bar{K} \cdot C L K) \cdot \mathrm{J}
\end{aligned}
$$

Operation:

1) $\mathrm{J}=\mathrm{K}=0$ : When both the inputs are in LOW state then the output follows its previous value. It means if the previous state is in the low state then the present output is also low and viceversa.

2) $\mathrm{J}=0, \mathrm{~K}=1$ : The output must be equal to ' 0 ' if the clock value is in the high state. If it is in the low state then the output simply follows its previous value.

3) $\mathrm{J}=1, \mathrm{~K}=0$ : In this case, the output is equal to ' 1 ' if the clock value is also high as shown in the truth table.

4) $\mathrm{J}=\mathrm{K}=1$ : When both the inputs are equal to ' 1 ' then the output is equal to the compliment of its previous value only when the clock value is also high.

The above equation i.e. equation 4 is the Boolean expression of the proposed circuit and the block diagram is shown in Fig.12 (a). The QCA implementation of the proposed circuit is shown in Fig. 12(b). In contrast with the circuit shown in the paper [8], the proposed scheme uses only 4 majority gates and 2 inverters. Also, the circuit is robust and more efficient in terms of a number of cells used. The area constraint is one of the major issues in designing digital circuits. This circuit is very promising related to the total area consumed.

\section{$3.41^{\text {st }}$ proposed structure of $T$ flip-flop}

Sequential circuits deploy flip flops as the memory element. In QCA, memory elements are formulated by using feedback paths. But according to the truth table 4 , the previous output is acting as an input to get the present value of output. And this is achieved by giving one clock cycle delay to Q. Fig.13 (b) displays the QCA equivalent of the 1st proposed scheme of T flipflop. It comprised of 3 majority gates and 2 inverters only. The output is simply the ex-or operation between $\mathrm{T}$ and the $\mathrm{Q}$. The output is obtained after one clock cycle delay.

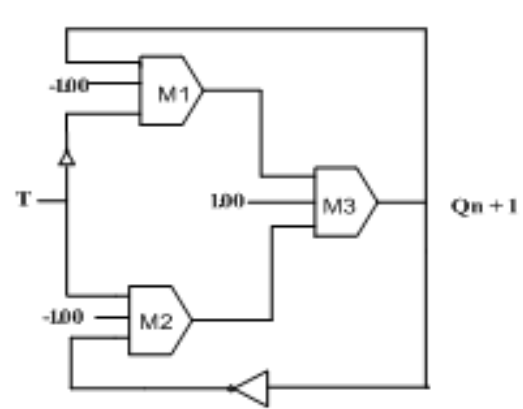

Fig. 13 (a) Majority schematic of proposed T flip flop

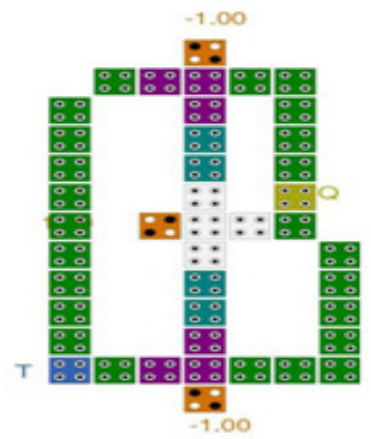

(b) Layout of proposed T flip flop 
International Journal of VLSI design \& Communication Systems (VLSICS) Vol.7, No.3, June 2016

\subsection{The second proposed T flip-flop structure}

As described for earlier designs in [7], [13], [21] and 1st proposed scheme, the output is feedback to the input side and due to this one clock cycle delay is produced in all the prior circuits. In order to avoid this, a novel structure of T flip-flop is proposed. In this structure, there is no need to give a feedback path from the output to the input. The clock signal is acting as the second input of the flip-flop. The clock used here is dual edge triggered type. The truth table for the operation is shown below.

Table 5: Dual edge triggered clock

Table 6: T flip-flop

\begin{tabular}{|l|l|l|}
\hline Clock $(\mathrm{t}-1)$ & Clock $(\mathrm{t})$ & Output \\
\hline 0 & 0 & Not changed \\
\hline 0 & 1 & Changed \\
\hline 1 & 0 & Changed \\
\hline 1 & 1 & Not changed \\
\hline
\end{tabular}

\begin{tabular}{|l|l|l|}
\hline $\mathrm{T}$ & $\mathrm{CLK}$ & $\mathrm{Q}_{n+1}$ \\
\hline 0 & 0 & $\mathrm{Q}_{n}$ \\
\hline 0 & 1 & $\mathrm{Q}_{n}$ \\
\hline 1 & 0 & $\overline{Q_{n}}$ \\
\hline 1 & 1 & $\overline{Q_{n}}$ \\
\hline
\end{tabular}

\section{Algorithm 2: Dual edge triggered T flip-flop}

Input $(\mathrm{A}, \mathrm{B}, \mathrm{C})$ where

$\mathrm{A}=\mathrm{T}$;

$\mathrm{B}=\mathrm{Clk}(\mathrm{t}-1)$;

$\mathrm{C}=\mathrm{Clk}(\mathrm{t})$;

Output $\left(\mathrm{Q}_{n}, \mathrm{Q}_{n+1}\right)$ where $\mathrm{Q}_{n}=$ previous output and $\mathrm{Q}_{n+1}=$ present output

Set input ((A, B, C) \& output $\left.\left(\mathrm{Q}_{n}\right)\right)$

$\mathrm{A}=0,1, \mathrm{X}$

$\mathrm{B}=0,1$;

$\mathrm{C}=0,1$;

$Q n=0$;

Set variable $\mathrm{i}=0$ to $\mathrm{n}$; (for the number of combination)

Transition $\left(\mathrm{Q}_{n+1}\right)$

\{

$\operatorname{If}(\operatorname{Clk}(\mathrm{t})=\operatorname{Clk}(\mathrm{t}-1)=0 / 1)$

$\mathrm{Q}_{n+1}=\mathrm{Q}_{n}$

else

$\mathrm{Q}_{n+1}=\mathrm{Q}_{n}$ for $(\mathrm{T}=0) ; Q_{n+1}=\bar{Q} n$ for $(\mathrm{T}=1) ;$

\}

Begin

\{

for $(i=0 ; i \leq 3 ; i++)$

\{

if $(\mathrm{C}=\mathrm{B})$;

$\mathrm{Q}_{n+1}=\mathrm{Q}_{n}$ for $(\mathrm{A}=\mathrm{X})$; 


\section{Else}

Call Transition

\}\}

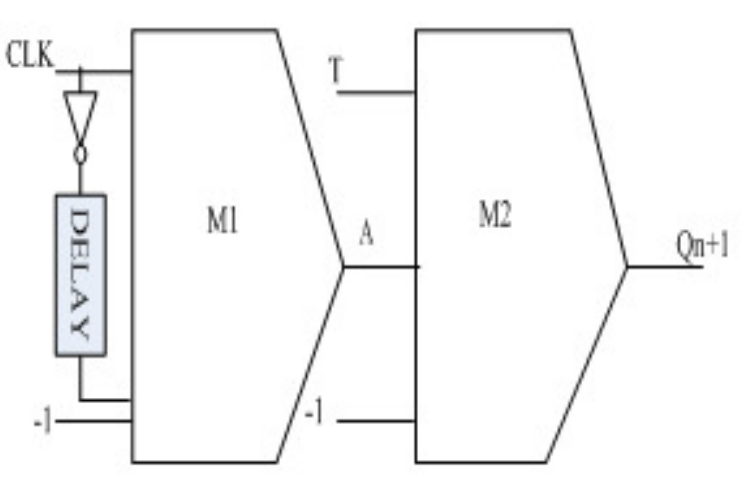

Fig.14: (a) $2^{\text {nd }}$ proposed schematic for $\mathrm{T}$ flip-flop

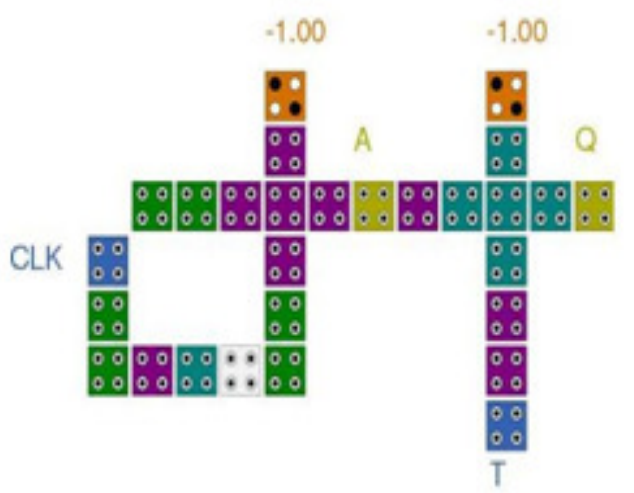

(b) QCA equivalent of T flip-flop

Let $\mathrm{Q}_{n}=0$ then the majority logic equation can be derived by observing the above block diagram as follows:

$Q_{n+1}=\mathrm{M}(\mathrm{T}, \mathrm{A}, 0)$,

where $\mathrm{A}=\mathrm{M}(\mathrm{CLK}, \mathrm{DCLK}, 0)$ and DLCK refers to delay in clock

The majority based equivalent diagram of the above equation is shown in Fig.14 (a). In contrast to the previous design, it consists of only 2 majority gates and 1 inverter. The advantage of this scheme is that there is no need to create a feedback path from the output side to the input side. Due to this, there is delay of 0.25 clock cycle in the circuit. The QCA equivalent diagram is shown in Fig. 14(b) which comprises of 28 cells occupying an area of $0.05 \mu \mathrm{m}^{2}$.

\section{APPLICATION OF PROPOSED T FLIP-FLOP}

\subsection{2-Bit Counter:}

Using T flip flop and some additional combinational logic circuits, any sequential logic design can be implemented. Counters and registers are the most prominent application in this digital era. Fig. 15 shows 2-bit counter (mod 4) by using T flip-flop. In this design, two T flip flops and four AND gates are used. The output of first $\mathrm{T}$ flip flop is applied as an input to the second $\mathrm{T}$ flip flop. The output Q0 toggles for every clock cycle. The output Q1 changes only when Q0 changes from 1 to 0 . Accordingly a 3-bit counter can also be designed as shown in Fig.16. The clock pulse is applied as a second input to the majority gate. The output of the first flip-flop is applied to second flip flop after performing an AND operation with the clock pulse. The output Q0 changes when the clock pulse remains high. 


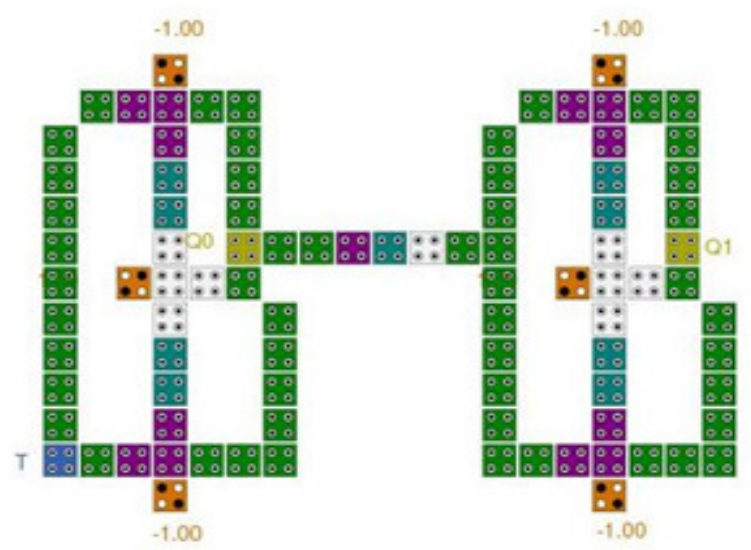

Fig.15 2-bit counter QCA layout

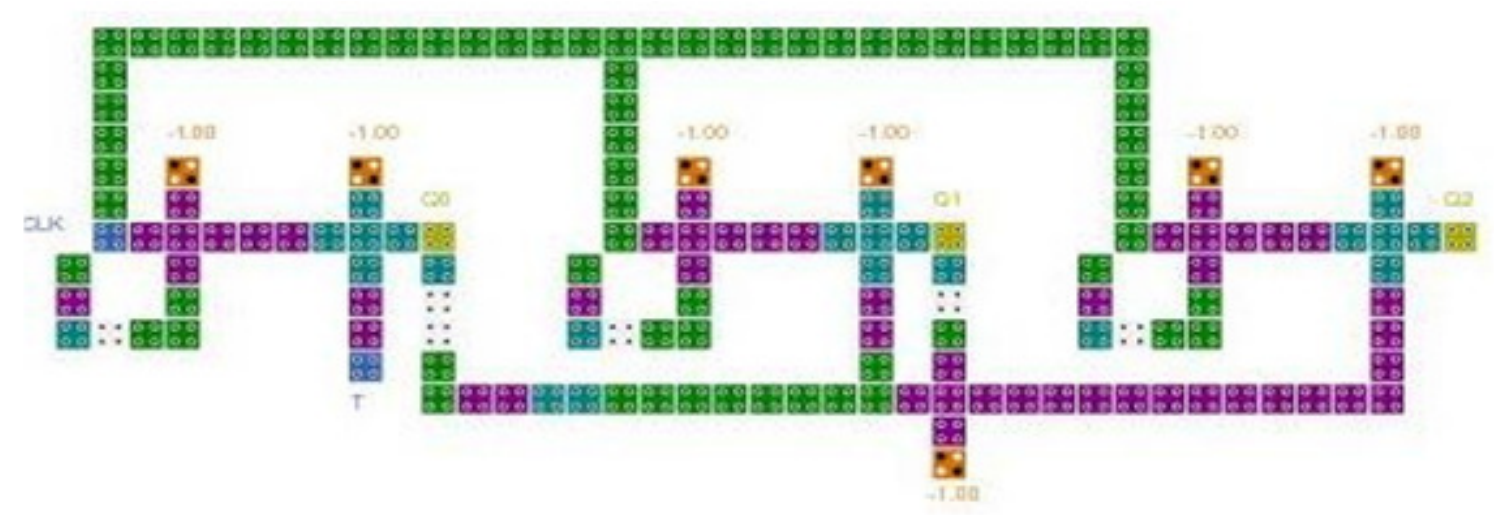

Fig.16 3-bit counter QCA block diagram

\section{SIMULATION RESULTS AND DISCUSSION}

In this section, the proposed methods are simulated and their correctness has been proved by using QCADesigner tool. This tool is a type of simulator which simulates the QCA logic components and reports the result in terms of the input-output state. Additionally the discussion of power dissipation is elaborated in this section. Based on the simulation result, it is clear that the proposed design works correctly.

\subsection{Power Dissipation Analysis:}

In QCA computations, only a few electrons are involved, it is susceptible to thermal issues. Therefore, power analysis is vital to model as an important parameter to analyze QCA design process along with errors. The most appropriate comprehension about it was studied well by J.Timler and C.S.Lent [15]. They have shown power dissipation during 3 events within QCA: clock power, cell-to-cell power gain, and power dissipation. With the help of these estimates, we can choose best alternative and parameters among many different designs. The power estimates computed on a per cell basis can be the sum total to obtain power dissipation for a QCA circuit. Each cell within a QCA circuit goes through the following three types of events: 1) to "depolarize" a cell, clock is going from low to high; 2) cells in previous clock zone called as "switching state"; and 3) clock changing from high to low, latching and holding the cell state to 
International Journal of VLSI design \& Communication Systems (VLSICS) Vol.7, No.3, June 2016

new state. Each of these events is associated with power loss [18].The keen interest in this discussion is that even during the first and third event the state of the cell would change or not, power dissipation is there due to clock changing only. This condition is analogous to the "leakage" power in CMOS circuits. And the power loss due to the second event is simply termed as "switching" power as it involves the cells actually changing their states. These two components are inversely related with each other. In QCA, a clock is used as an avenue to lower and raise energy barriers and provide power to the system.

\subsection{Kink energy:}

In the study of power dissipation the concept of kink energy is useful to mention. When the system is stuck at any metastable state, a kink is said to occur. The kink energy $\mathrm{E}_{k}$ is the energy required to excite the system from the ground state to the first excited state. It must be greater than $\mathrm{k}_{B} \mathrm{~T}$ to set apart a bit value from the thermal environment where $\mathrm{T}$ is temperature in degrees Kelvin and $\mathrm{k}_{B}$ is Boltzmann's constant. In [15] they have proved that to defend against kink the number of QCA cells i.e. $\mathrm{N}$ in the longest line must be less than $e^{\frac{E k}{K b T}} \cdot$ To do this work a novel probabilistic modelling tool called as QCAPro is developed by the authors in [16]. To do the estimation of highly spurious cells in QCA circuit, this technique uses a fast approximation analysis. Since kink energy relates itself inversely with steady state polarization error and directly with power dissipation so it is required to choose an optimal value of kink energy. In this paper, the power dissipation analysis of first proposed $T$ flip-flop is discussed only and then it is compared with the existing MOSFET technologies. The same approach can be applied to the much complex circuits as well. According to the analysis observed using QCAPro, we can choose the best alternative among the available technologies to design a circuit. By varying the value of kink energy to three different values, the observed power is shown in Table 7.

Table 7 Comparative analysis of power dissipated for three different values of kink energy

\begin{tabular}{|c|c|c|c|}
\hline \multirow[t]{2}{*}{ Parameters } & \multicolumn{3}{|c|}{$\mathrm{E}_{k}=() \mathrm{meV} \times 10^{-2}$} \\
\hline & 0.5 & 1.0 & 1.5 \\
\hline Max Kink Energy & $0.00148 \mathrm{Ek}$ & $0.00148 \mathrm{Ek}$ & $0.00148 \mathrm{Ek}$ \\
\hline Max Energy Diss of Circuit & 0.13017 & 0.14150 & 0.15831 \\
\hline Max Energy Diss Vector & 20 & 20 & 20 \\
\hline Avg Energy Diss of Circuit(over all vector points) & 0.07162 & 0.09099 & 0.11506 \\
\hline Max Energy Diss vector & 0.00645 & 0.00622 & 0.00617 \\
\hline Min Energy Diss of Circuit & 02 & 31 & 20 \\
\hline \multirow[t]{2}{*}{ Min Energy Diss Vector } & 0.01435 & 0.04176 & 0.07356 \\
\hline & 00 & 00 & 00 \\
\hline Avg Leakage Energy Dissipation(over all vector pairs) & & & \\
\hline \multirow[t]{2}{*}{ Avg Switching Energy Dissipation(over all vector pairs) } & 0.01496 & 0.04251 & 0.07426 \\
\hline & 0.05666 & 0.04848 & 0.04079 \\
\hline
\end{tabular}

From the above table, we can see that average leakage energy is increasing with increase in kink energy and vice versa for average switching energy. We, therefore, limited to choose an optimum value of kink energy for proper operation of the circuit. The power dissipation is very small as 
International Journal of VLSI design \& Communication Systems (VLSICS) Vol.7, No.3, June 2016

compared to the conventional Mosfet technologies. Thus, our proposed circuit is meeting the current demand for the immediate present designs in other paper.

\subsection{Thermal layout:}

The researchers in [17] have evaluated the consumed power of the circuit using QCAPro which reveals the design at three different tunnelling energy levels $(0.5 \mathrm{Ek}, 1 \mathrm{Ek}, 1.5 \mathrm{Ek})$ at $2 \mathrm{~K}$ temperature. The thermal layout of the T flip-flop is shown in Fig.17. The cells in dark colour indicate that it is dissipating higher power. And it is called as the thermal hotspot. The total power dissipation is divided into leakage and switching energies as shown in Table 7 . The cells at the input side are dissipating very low power.

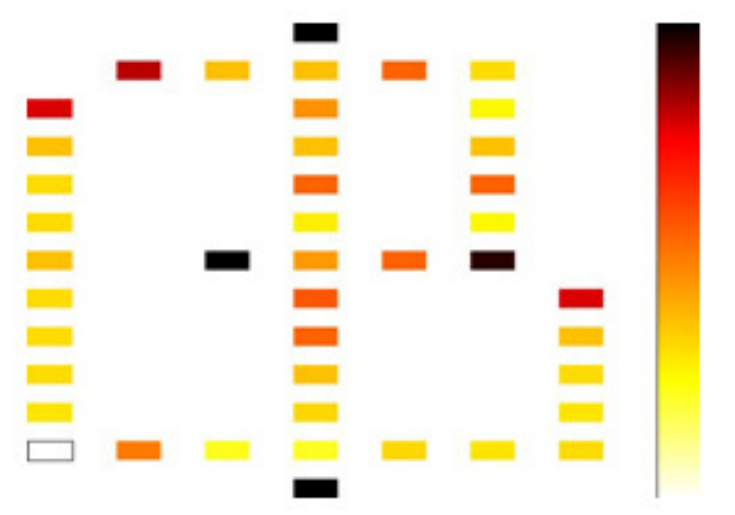

Fig.17 Thermal layout of proposed T flip-flop

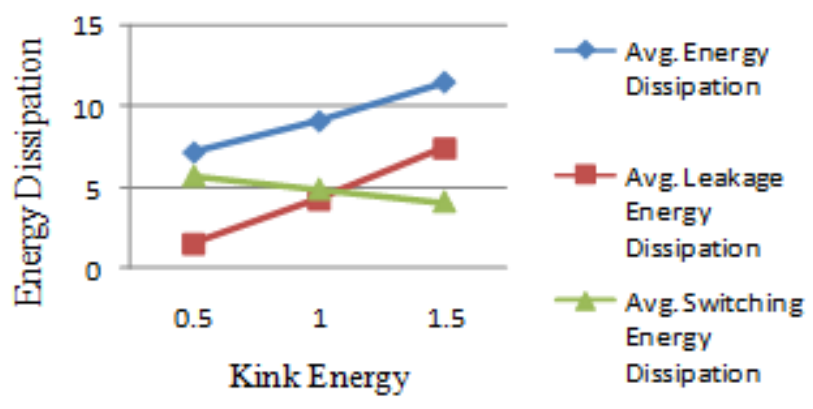

Fig. 18: Graph between energy dissipation and kink energy

\subsection{Variation of energy dissipation with kink energy:}

As the value of kink energy is increased gradually from 0.5 to 1.5 , the average energy dissipation factor goes on increasing as shown in Fig.18. It means it varies linearly with kink energy. The average leakage energy also follows a linear relationship with kink energy. Hence, an optimum value of kink energy is chosen to operate the circuit so as to deliver the advantage correctly. In contrast to this, the average switching energy dissipation shows a decreasing ramp with increasing value of kink energy. It is, therefore, there is a trade-off between the power dissipation and the value of kink energy. 
International Journal of VLSI design \& Communication Systems (VLSICS) Vol.7, No.3, June 2016

\subsection{Results and Discussion:}

A. Output waveform of proposed level triggered JK flip-flop

The output of Fig.12 (b) is verified correctly after seeing the simulation results. The results are valid only when the clock input is held high. Therefore, the output does not go to SET state even when $\mathrm{J}$ and $\mathrm{K}$ are in the high state. It is worth mentioning to revise again that it occurs because the clock pulse is not high in that input condition. Hence, the circuit changes its state only when the clock is enabled. There is a delay of one clock cycle between input and output which is superior as compared to the design in [20].

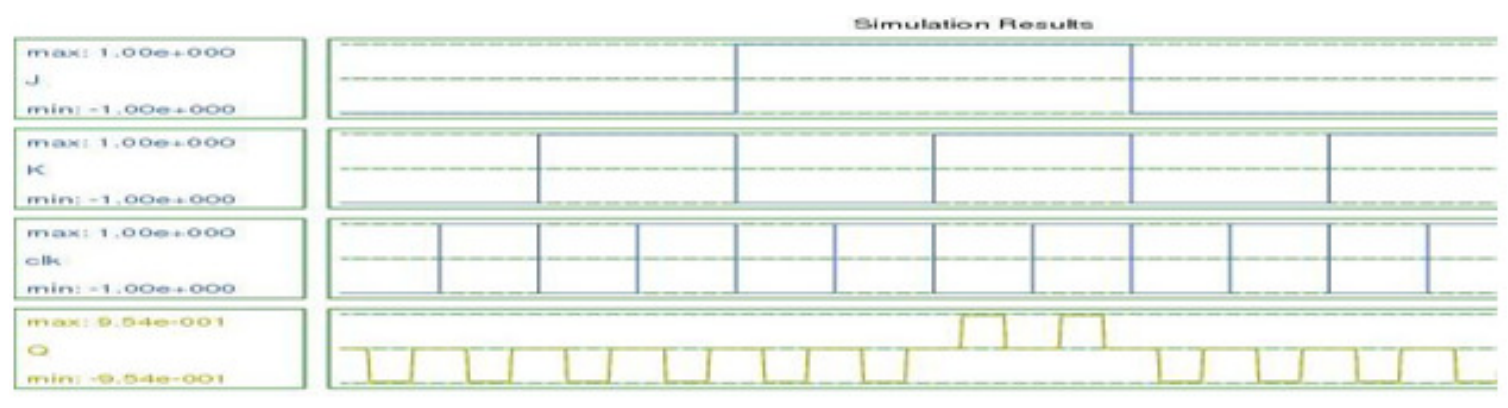

B. Output waveform of 1 st proposed T flip-flop

This is the output of first propose design of T flip-flop shown in fig. 13(b). The output toggles after one clock cycle delay when $\mathrm{T}$ becomes high. The number of cells reduced to a small extent as compared with reference [21]. The disadvantage of this circuit is that there is no control of clock in its operation. Thus, a more conventional approach is required

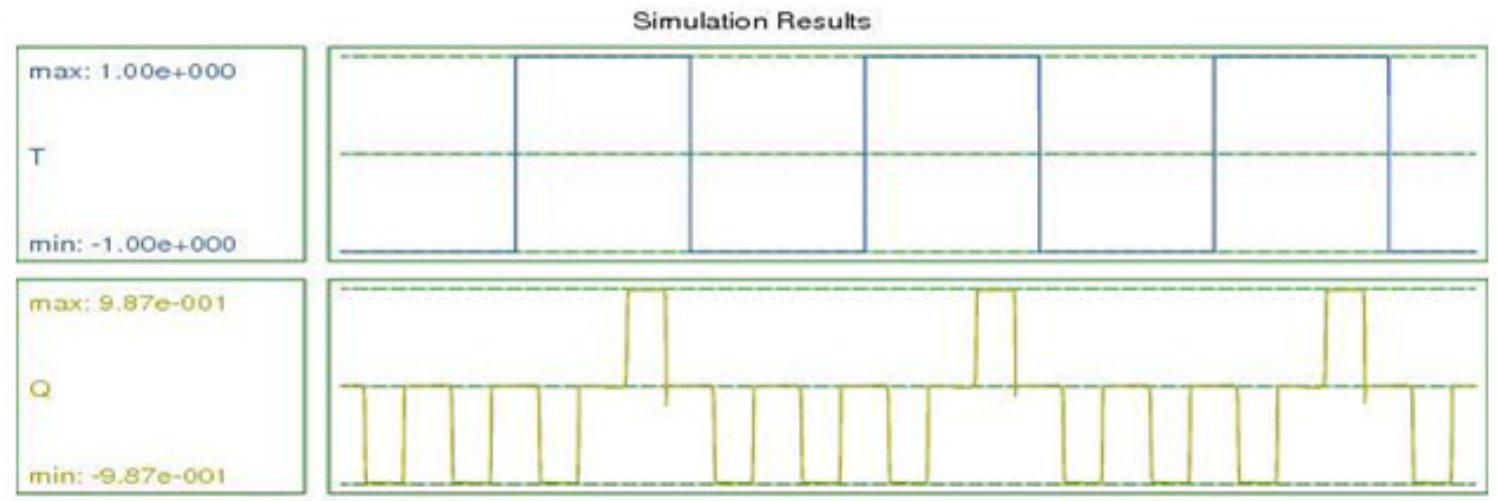

C. Output waveform of 2nd proposed T flip-flop

This is the output waveform of 2 nd proposed $\mathrm{T}$ flip-flop shown in fig.14 (b). This is a novel concept of implementing $\mathrm{T}$ flip-flop. This circuit has many advantages as compared to the existing designs till now. Firstly it does not require creating a feedback path from the output to the input side. Secondly, the number of cells used is very less and hence the area occupied is only about $0.05 \mu \mathrm{m}^{2}$. And at last, we can see the output is obtained even before the one cycle of clock completes. Hence, delay between input and output is even less than one cycle. The output toggles only when the clock makes a transition from 1 to 0 or 0 to 1 as shown in the simulation result. 
International Journal of VLSI design \& Communication Systems (VLSICS) Vol.7, No.3, June 2016

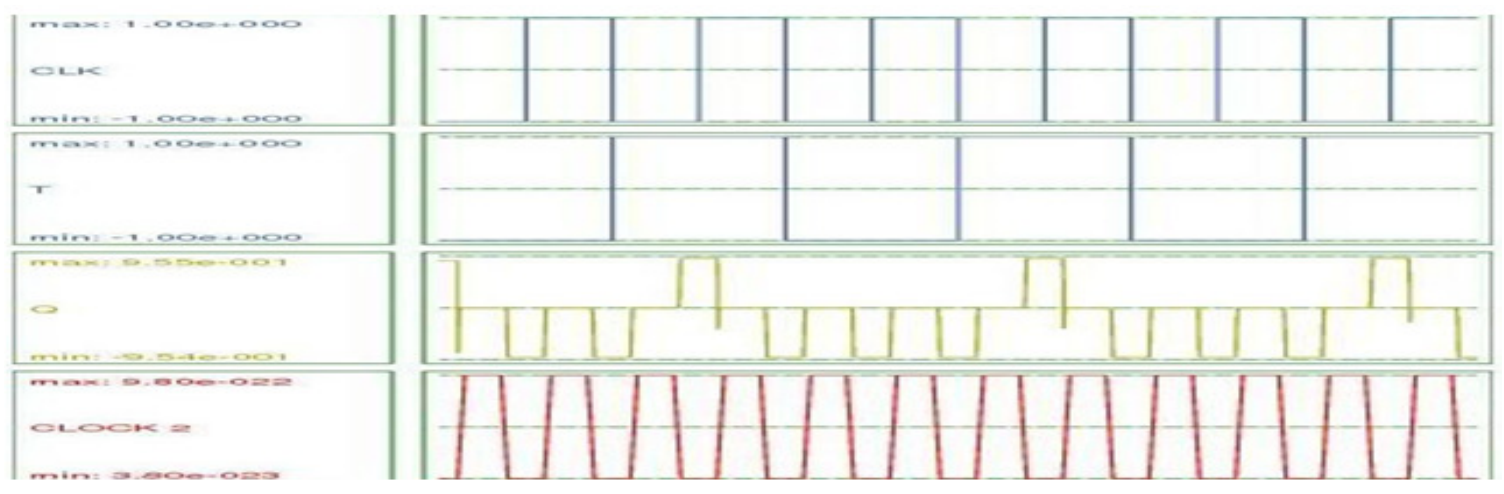

D. Output waveform of 2-bit counter

This is the output of 2-bit counter shown in Fig.15.This is a $\bmod (\mathrm{N})$ counter where $\mathrm{N}=$ number of states obtained and given as:

$\mathrm{N} \leq 2 \mathrm{n}$; where $\mathrm{n}=$ a number of flip flops.

Therefore, we obtained four numbers of states at the output as the number of the flip-flop used is two.

This counter uses only 92 cells to give valid output and the delay is only two clock cycle.
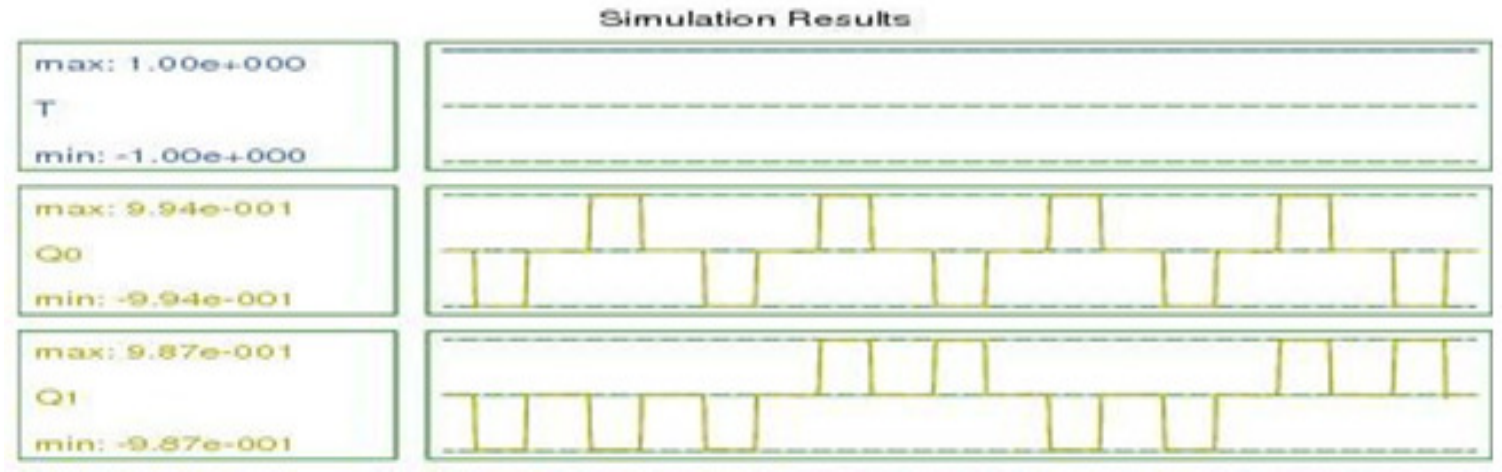

Table 8 Comparison of different implementations

\begin{tabular}{|c|c|c|c|}
\hline & Number of cells used & $\begin{array}{l}\text { Covered Area } \\
\left(\mu m^{2}\right)\end{array}$ & Input to output delay \\
\hline $\mathrm{JK}$ in [21] & 120 & 0.22 & 2 clock cycle \\
\hline $\mathrm{JK}$ in [22] & 80 & 0.12 & 1.5 clock cycle \\
\hline $\mathrm{T}$ in $[23]$ & 66 & 0.06 & 1.5 clock cycle \\
\hline $\operatorname{Tin}[22]$ & 108 & 0.20 & 1.5 clock cycle \\
\hline 2 -bit counter in [21] & 328 & 0.62 & 3 clock cycles \\
\hline 3 -bit counter in $[21]$ & 616 & 1.2 & 5 clock cycles \\
\hline Proposed JK & 54 & 0.09 & 1.5 clock cycle \\
\hline $1^{\text {st }}$ proposed T & 43 & 0.04 & 1 clock cycle \\
\hline $2^{\text {nd }}$ proposed $\mathrm{T}$ & 28 & 0.05 & 0.25 clock cycle \\
\hline Proposed 2-bit counter & 92 & 0.11 & 2 clock cycle \\
\hline Proposed 3-bit counter & 159 & 0.24 & 3 clock cycle \\
\hline
\end{tabular}

The implementation of JK flip-flop is shown in Fig.12 (b) uses only 54 cells which improve around $32 \%$ as compared to the previous designs. The second proposed method of $\mathrm{T}$ flip-flop in which output toggles when input $\mathrm{T}$ is high. It, therefore, gives its output after one clock delay as 
International Journal of VLSI design \& Communication Systems (VLSICS) Vol.7, No.3, June 2016

its present output depends on the value of the previous one. The third proposed model includes the effect of the clock on the output of T flip-flop. In this case, the output toggles only when the clock input goes from 1 to 0 . This approach is based on a new concept of avoiding feedback from output to the input side. The fourth QCA model for T flip flop is just an approach used by Xiao Kou Yang in [21].

\section{CONCLUSIONS}

The flip-flop is the backbone circuit to design sequential logical functions. This paper has proposed falling edge triggered and positive level triggered methods of designing flip flops. Later on, these designs are further expanded to obtain 2-bit and 3-bit counter circuits which are a very demanding application in the digital world. Simulation results approve the existence of proposed designs. The new approach of designing $\mathrm{T}$ flip-flop is attractive in terms of a number of cells used and the concept of removing feedback paths. But still many questions on sequential circuits is required to explore and answer so as to design QCA at large scale application.

\section{REFERENCES}

[1] P.D. Tougaw, C.S. Lent (1994) "Logical devices implemented using quantum cellular automata", Appl. Phys. Vol. 75, pp.1818-1824.

[2] C. Lent, P. Tougaw, and W. Porod (1994) "Quantum cellular automata: the physics of computing with arrays of quantum dot molecule", Physics and Computation Proceedings Workshop, vol. 94, , pp. 5-13.

[3] Amir Mokhtar Chabi, Samira Sayedsalehi, Shaahin Angizi, and Keivan Navi (2014) "Efficient exclusive-or and multiplexer circuit based on a nanoelectric-compatible deigning approach", Hindawi publishing Corporation, pp. 1-9.

[4] Shaahin Angizi, Esam Alkaldy, Nader Bagherzadeh, and Keivan Navi (2014) "Novel robust single layer wire crossing approach for exclusive-or sum of products logic design with QCA", Journal Of Low Power Electronics, Vol. 10, pp.259-271.

[5] Kondwani Makanda, Jun-Cheol Jeon (2013) "Improvement of quantum dot cellular automata decoder uing inverter chain", Advanced Science and Technology Letters, Vol.29, pp.227-229,.

[6] Mohammad Reza Raaei Moghaddam, Ali Akbar Khazaei, Davoud Bahrepour (2014) "Designing an improved asynchronous memory based on qca", 9th Symposium on Advances in Science and Technology, Mashad,Iran..

[7] Mostafa Abdollahian Dehkordi, Abbas Shahini Shamsabadi, Behrouz Shahgholi Ghahfarokhi ,Abbas Vafaei (2011) "Novel RAM cell designs based on inherent capabilities of quantum-dot cellular automata", Microelectronics Journal 42 pp.701-708.

[8] Lin-rong XIAO, Xie-xiong CHEN, Shi-yan YING (2012) "Design of dual-edge triggered flip flops based on quantum-dot cellular automata", Journal of Zhejiang University-SCIENCE C (Computers \& Electronics), volume 13(5) pp.385-392.

[9] W.Wang, K.Walus, J.Julien (2003) "Quantum dot cellular automata adders", Third IEEE Conference on Nanotechnology, IEEE-NANO, pp. 461-464.

[10] Lent, C. S. and B. Isaksen (2003) "Clocked Molecular Quantum-Dot Cellular Automata", IEEE Transactions on Electron Devices, Vol. 50, No. 9, pp. 1890-1895.

[11] Lent, C. S. and P. D. Tougaw (1997) "A device architecture for computing with quantum dots" ,Proc. Of the IEEE, Vol. 85, pp. 541-557.

[12] K.Navi, M.Moayeri, R.Faghih Mirzae, O.Hashemipour, B.Mazloom Nezhad (2009) “Two new low power full adder based on majority-not gate", Microelectronics Journal, 40 (1) pp.126-130.

[13] Abbas Shahini Shamsabadi, Behrouz Shahgholi Ghahfarokhi, Kamran Zamanifar, Naser Movahedinia (2009) "Applying inherent capabilities of quantum-dot cellular automata to design:D flip- flop case study”, Journal of Systems Architecture 55 pp.180-187. 
International Journal of VLSI design \& Communication Systems (VLSICS) Vol.7, No.3, June 2016

[14] J. Huang, M. Momenzadeh, F. Lombardi (2007) "Design of sequential circuits by quantum-dot cellular automata" Microelectronics Journal 38 pp.525-537.

[15] John Timler and Craig S. Lent (2002) "Power gain and dissipation in quantum-dot cellular automata", Journal of applied physics, volume 91, issue 2, pp. 823-831 .

[16] Jing Huang, Fabrizio Lombardi (2008) "Design and test of digital circuits by quantum dot cellular automata", Boston, MA .

[17] Saket Sivastava, Arjun Asthana, Sanjukta Bhanja, Sudeep Sarkar (2011) "QCAPro- An Error-power estimation tool for qca circuit design", IEEE International Symposium of Circuits and Systems (ISCAS), Rio de Janeiro, pp. 2377 - 2380.

[18] Shadi Sheikhfaal, Shaahin Angizi, Soheil Sarmadi, Mohammad Hossein Moaiyeri (2015) "Designing efficient qca logical circuits with power dissipation analysis", Microelectronics Journal, 46, pp. 462471.

[19] Saket Srivastava, Sudeep Sarkar, anjukta Bhanja (2009) "Estimation of upper bound of power dissipation in qca circuits", IEEE Transactions on Nanotechnology, volume 8 (1), pp.116-127.

[20] Bibhash Sen, Mrinal Goswami, Subhra Mazumdar, Biplab K Sikdar (2015) "Towards modular design of reliable quantum-dot cellular automata logic circuit using multiplexers", Computers and Electrical Engineering, Elsevier vol.45, pp.42-54.

[21] Xiaokou Yang, Li Cai, Xiaohui Zhao, Nansheng Zhang (2010) "Design and simulation of sequential circuits in quantum dot cellular automata: falling edge-triggered flip flop and counter study", Microelectronics Journal 41, pp. 56-63.

[22] Lee Ai Lim, Azrul Ghazali, Sarah Chan Tji Yan, Chau Chein Fat (2012) "Sequential circuit design using quantum-dot cellular automata (QCA)", IEEE Conference, Kajang, Selangor, Malaysia 978-14673-3119.

[23] Mohammad Torabi (2011) "A new architecture for T flip flop using qca", 3rd Asia Symposium on Quality Electronic Design 978-1-4577-0144, pp. 296-300.

\section{AUTHORS}

Shraddha Pandey received the B. Tech degree in Electronics and Communication from the Bhabha Institute of Technology, Kanpur affiliated to AKTU, Lucknow U.P., India, in 2012. She is currently pursuing her M. Tech. in Microelectronics from IET, Lucknow. Her research interest is in Low power VLSI design, Digital design and system, Quantum-dot cellular automata and nanoelectronics.

Sonali Singh received the B. Tech degree in Electronics and Communication form the Bundelkhand Institute of Engineering And Technology, Jhansi, U.P., India in 2013. She is currently pursuing towards her M. Tech. in Microelectronics from IET, Lucknow. Her research interest is in VLSI design, Digital circuits and system, Quantum-dot cellular automata and Nanoelectronics.

Dr. Subodh Wairya received B. Tech (1993), M.Tech and Ph.D (2012) from HBTI, Kanpur, Jadavpur University, Kolkata and MNNIT Allahbad, India, respectively. His research area are High speed network, Low power VLSI, Nanoelectronics. Currently, he is an Professor at IET, lucknow (from 6 May 1996-Present).He has also served as Scientist "B" Adhoc (One Year) at DRDO, Lucknow during January, 1995-January, 1996 and Graduate Engineer under Consultancy Project at HAL, Lucknow during From January, 1994-January, 1995 (one year). Also he is one of the authors of a book
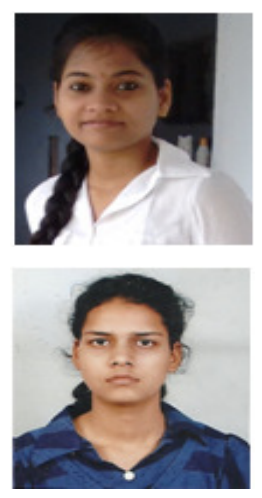
entitled "A Simplified Approach to Telecommunication and Electronic Switching Systems" by C.B.L. Srivastava, Neelam Srivastava \& Subodh Wairya Published by Dhanpat Rai and Company in the year 2006. 